\title{
Mobile learning for delivering health professional education (Protocol)
}

Tudor Car L, Riboli-Sasco EF, Marcano Belisario JS, Nikolaou CK, Majeed A, Zary N, Car J

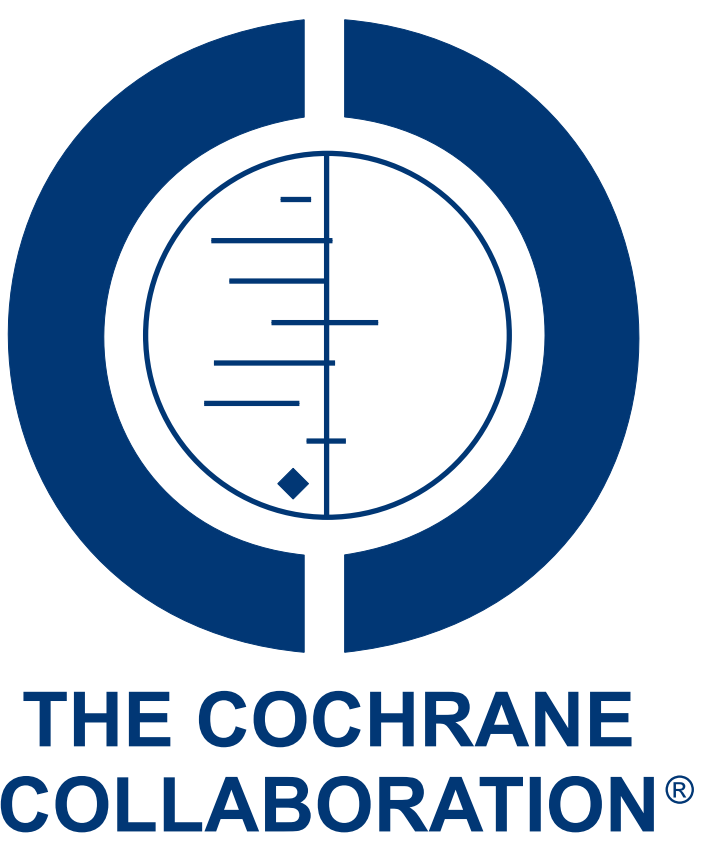

This is a reprint of a Cochrane protocol, prepared and maintained by The Cochrane Collaboration and published in The Cochrane Library 2015, Issue 9

http://www.thecochranelibrary.com

\section{WILEY}

Mobile learning for delivering health professional education (Protocol)

Copyright $\odot 2015$ The Cochrane Collaboration. Published by John Wiley \& Sons, Ltd. 
TABLE OF CONTENTS

HEADER . . . . . . . . . . . . . . . . . . . . . . . . . . . . . . . . . . . . 1

ABSTRACT . . . . . . . . . . . . . . . . . . . . . . . . . . . . . . . . . . . . . . . 1

BACKGROUND . . . . . . . . . . . . . . . . . . . . . . . . . . . . . . . . . . . .

OBJECTIVES . . . . . . . . . . . . . . . . . . . . . . . . . . . . . . . . . . . . . . .

METHODS . . . . . . . . . . . . . . . . . . . . . . . . . . . . . . . . . . . . . .

ACKNOWLEDGEMENTS . . . . . . . . . . . . . . . . . . . . . . . . . . . . . . . . . . . . . . . .

REFERENCES . . . . . . . . . . . . . . . . . . . . . . . . . . . . . . . . . . . . . . .

APPENDICES . . . . . . . . . . . . . . . . . . . . . . . . . . . . . . . . . . . . . 9

CONTRIBUTIONS OF AUTHORS . . . . . . . . . . . . . . . . . . . . . . . . . . . . . . . . . . . . . . .

DECLARATIONS OF INTEREST . . . . . . . . . . . . . . . . . . . . . . . . . . . . . . . . 13

SOURCES OF SUPPORT . . . . . . . . . . . . . . . . . . . . . . . . . . . . . . . . . . . . . . . . . . 


\title{
Mobile learning for delivering health professional education
}

\author{
Lorainne Tudor Carr $^{1}$, Eva F Riboli-Sasco ${ }^{2}$, José S Marcano Belisario ${ }^{3}$, Charoula K Nikolaou ${ }^{4}$, Azeem Majeed ${ }^{2}$, Nabil Zary ${ }^{5}$, Josip Car \\ $4,6,7$ \\ ${ }^{1}$ School of Public Health, Imperial College London, London, UK. ${ }^{2}$ Department of Primary Care and Public Health, Imperial College \\ London, London, UK. ${ }^{3}$ Global eHealth Unit, Department of Primary Care and Public Health, School of Public Health, Imperial \\ College London, London, UK. ${ }^{4}$ Lee Kong Chian School of Medicine, Nanyang Technological University, Singapore, Singapore. \\ ${ }^{5}$ Department of Learning, Informatics, Management and Ethics (LIME), Karolinska Institutet, Stockholm, Sweden. ${ }^{6}$ Global eHealth \\ Unit, Department of Primary Care and Public Health, School of Public Health, Imperial College London, London, UK. ${ }^{7}$ Department \\ of Family Medicine, Faculty of Medicine, University of Ljubljana, Ljubljana, Slovenia
}

Contact address: Josip Car, Lee Kong Chian School of Medicine, Nanyang Technological University, Singapore, Singapore. josip.car@imperial.ac.uk. josip.car@ntu.edu.sg.

Editorial group: Cochrane Tobacco Addiction Group.

Publication status and date: New, published in Issue 9, 2015.

Citation: Tudor Car L, Riboli-Sasco EF, Marcano Belisario JS, Nikolaou CK, Majeed A, Zary N, Car J. Mobile learning for delivering health professional education. Cochrane Database of Systematic Reviews 2015, Issue 9. Art. No.: CD011861. DOI: 10.1002/14651858.CD011861.

Copyright (C) 2015 The Cochrane Collaboration. Published by John Wiley \& Sons, Ltd.

\begin{abstract}
A B S T R A C T
This is the protocol for a review and there is no abstract. The objectives are as follows:

The objective of this review is to evaluate the effectiveness of mLearning educational interventions for delivering pre-registration and post-registration healthcare professional education. We will primarily assess the impact of these interventions on students' knowledge, skills, professional attitudes and satisfaction.
\end{abstract}

\section{B A C K G R O U N D}

\section{Description of the condition}

The current global shortage of doctors, nurses, midwives, and allied healthcare workers has been estimated at 7.2 million (Campbell 2013), and is expected to reach 12.9 million by 2035 (WHO 2013). This shortage is more apparent in low-and-middle-income countries where the migration of many students and fully qualified workers further reduces already limited resources (Kuehn 2007; Marchal 2003).

This shortage and disproportionate distribution of health workers worldwide (Chen 2010) can be aggravated by the inadequacy of training programmes (in terms of content, organisation and delivery) to equip trainees with the skills, competencies and experience needed to face the realities of the settings in which they are to work (Frenk 2010). It has therefore become essential to focus effort and resources on developing and implementing strategies that can lead to an increase in both the number of health care workers and the quality and relevance of their training (Global Health Workforce Alliance 2015; WHO 2011). The increased use of information and communication technologies for educational purposes (i.e., eLearning) has been recognised as one of the key strategic platforms to build strong health education and training systems (Crisp 2008). 


\section{Description of the intervention}

This protocol for a systematic review is part of a series of systematic reviews that will evaluate the efficacy of different types of eLearning interventions for delivering pre- and post-registration health professional education.

eLearning is a broad construct describing a wide range of teaching and learning strategies that are exclusively based on the use of electronic media and devices as training, communication and interaction tools (Sangrà 2012). eLearning enables distant learning which could address the problems of shortage of healthcare manpower, educators and limited resources by overcoming geographical or temporal constraints and thereby reducing the cost and the inconvenience of travelling. If used in mixed delivery mode (i.e., in combination with traditional educational strategies such as classroom face-to-face teaching) it should be referred to as 'Blended learning'.

eLearning consists of various types of interventions that can be characterised in different ways: according to the delivery tools, content, learning objectives, pedagogical approaches or settings of delivery. We classified eLearning interventions into six main categories based on the technology as well as the pedagogical approach used:

1. offline computer-based eLearning;

2. online computer-based eLearning;

3. digital game-based learning;

4. massive open online courses;

5. psychomotor skills trainers, virtual reality environments and virtual patient simulations; and

6. mobile learning (mLearning)

In this protocol, we will focus on mLearning.

There is no uniformly accepted definition of mLearning. This lack of consensus not only arises from the rapid evolution of the field, but also from the ambiguity of the term mobile. This term can be used to describe either the technology used for delivering the educational content (e.g., through a smartphone or tablet), or the situational context in which learning takes place (e.g., on the way back home) (Hashemi 2011). Earlier definitions of mLearning were technocentric and only focused on the types of devices used, whereas more recent definitions of mLearning give more weight to the learner and the context in which the learning takes place. In the Handbook of Mobile Learning, mLearning was defined as "learning across multiple contexts, through social and content interactions, using personal electronic devices" (Crompton 2013). The latter definition, however, creates ambiguity around the type of devices, particularly given the number of personal consumer devices, such as laptops, that are currently available in the market.

To avoid such ambiguity, we will consider mLearning in health professional education as any intervention using handheld, mobile devices connected through wireless connections to deliver educational content to pre- and post-registration students in order to extend the reach of learning and teaching beyond physical space and distance. mLearning is increasingly used in health profession- als' education before qualification (pre-registration) as well as after it (post-registration) e.g., as part of speciality training, continuous medical education or continuous personal development. In this review, we intend to collate and present the evidence on the use of mLearning in pre- and post-registration health professionals' education. We will consider eligible studies on candidates for, and holders of, the qualifications listed in the Health Field of Education and Training of the International Standard Classification of Education. We combine both the technocentric and the learnercentred approaches by defining handheld, mobile devices as being "small, autonomous, and unobtrusive enough to accompany us in every moment of our every-day life” (Trifonova 2003). Arguably considering the 'power' of modern hand-held devices - many if not all of the eLearning interventions could be foreseeably delivered via mLearning.

\section{How the intervention might work}

Mobile technologies have been described as "ubiquitous/spontaneous, portable, blended, private, interactive, collaborative and immediate" (Ozdamli 2011). Such characteristics make their use in professional health education promising (Sharples 2002).

Through their connectivity capabilities, mobile devices could enable the delivery of educational content without any geographical or temporal constraints, thus extending the reach of and increasing the access to education delivery (Hashemi 2011; O’Malley 2005). The potential reach of mLearning may be of particular interest in low- and middle-income countries and remote areas with poor transport infrastructure and teaching facilities (Ally 2009). Some of the different modes of communication that mobile devices can support, (e.g., short message service (SMS), multimedia messaging service (MMS), applications or "apps", or podcasts) do not require complex infrastructures for their effective deployment.

While access to computers, land line phones or wired Internet is still restricted, the low and decreasing cost of most mobile devices has allowed for their spread on a global scale, including to the most deprived settings. In developing countries, there were more than 80 mobile subscriptions for every 100 inhabitants in 2013 (ITU 2015), and in high-income countries such as the USA and the UK there are now more than one subscription per person (World Bank 2013). A recent report by the Pew Research Center estimated that $68 \%$ of adults in the United States connect to the Internet with mobile devices (Pew Research Center 2014).

By relying on a technology that is widely used and available, mLearning interventions require a reduced investment and may facilitate its adoption by the learners. In addition they take advantage of the familiarity of the students with such devices, thus reducing the costs of training as well as the risk of rejection.

As the use of personal digital assistants (PDAs), mobile phones apps and other mobile technologies increase in healthcare settings, early exposure to mLearning during training of health profession- 
als could promote better familiarity with such devices, for their use in future professional practice.

\section{Possible disadvantages and risks of the intervention}

As with other fields of eLearning, mLearning has quickly grown in less than a decade, showing great potential in terms of reducing costs and increasing adaptability and portability of learning. However, it brings along several technical challenges including, the limited screen size and memory of the devices. There is also a risk of distraction and sudden obsolescence. mLearning could also introduce a number of social and educational challenges, particularly relating to accessibility by disadvantaged populations. Another issue related to the use of mLearning for health professional education could be the "digital divide" which refers to the gaps and differences in access and use of information and communication technologies arising both among but also within countries (OECD 2001).

The mobility associated with mLearning, as compared to other forms of eLearning, also raises concern due to the increased risk of distractions associated to mobile learning environments. This calls for a specific and appropriate pedagogical approach adapted to the devices as well as to the context in which the learning is to take place (Motiwalla 2007).

\section{Why it is important to do this review}

Past reviews have underlined the potential of eLearning interventions but also stressed the need for further research and reviews on the topic (Childs 2005; Cook 2010; Feng 2013; George 2014; Lahti 2014; Rasmussen 2014; Rosenberg 2003; Rowe 2012). This is mainly due to the limited scope of existing evaluations in terms of outcomes (user enjoyment and satisfaction rather than knowledge and skills acquisition), duration (short-term rather than longterm), professional field (nurses, medical education), educational context (mostly high income countries), technology used (nonnetworked, computer-based; networked; virtual reality) and broad range of measurement instruments and units used (from multiplechoice to observational methods).

Our review will address the existing gaps by:

- updating the fast growing body of evidence on the topic of mLearning, and inform and guide its future use in health professional education;

- focusing on mLearning interventions across various professional fields of health sciences education at the pre- and post-registration levels;

- evaluating the impact of such interventions on students' knowledge, skills, satisfaction and professional attitudes;
- including evidence from high as well as low- and middleincome countries;

- being integrated in a series of reviews which will provide a systematic approach to the multiple uses and application of eLearning in terms of channels (online and offline computers, simulated environments, games and blended learning) and training stages (pre- and post-registration students in continuing professional development).

\section{O B J E C T IVES}

The objective of this review is to evaluate the effectiveness of mLearning educational interventions for delivering pre-registration and post-registration healthcare professional education. We will primarily assess the impact of these interventions on students' knowledge, skills, professional attitudes and satisfaction.

\section{METHODS}

\section{Criteria for considering studies for this review}

\section{Types of studies}

We will include randomised controlled trials (RCTs) and clusterRCTs.

We will also include RCTs with unclear or high risk of bias for sequence generation. If meta-analysis of included studies is feasible and appropriate, we will include all RCTs regardless of their sequence generation bias rating. However, we will also conduct sensitivity analyses excluding those at unclear or high risk of bias, to examine the robustness of the meta-analysis results to methodological limitations of the included studies. We will exclude crossover trials due to high likelihood of carry-over effect.

\section{Types of participants}

We will include studies with participants who are enrolled either in:

- a pre-registration, undergraduate, health-related university degree, or a basic health-related vocational training programme. We will define pre-registration, undergraduate educational or basic vocational training as any type of study leading to a qualification that: (i) is recognised by the relevant governmental or professional bodies of the country where the studies were conducted; and (ii) entitles the qualification-holder to apply for entry level positions in the healthcare workforce. For this reason, graduate medical education courses from the USA as well as 
other countries with graduate medical education courses will be included in this category; or

- a post-registration health professional educational programme, defined as any type of study after a qualification which is recognised by the relevant governmental or professional bodies that enables the qualification holder entry into or continuation of work in the healthcare workforce in a more independent or senior role.

We will include candidates for, and holders of, the qualifications listed in the Health Field of Education and Training of the International Standard Classification of Education (UIS 2012), except students of traditional, alternative and complementary medicine. We will therefore include students from the following categories: dental studies, medicine, nursing and midwifery, medical diagnostic and treatment technology, therapy and rehabilitation, and pharmacy.

Participants will not be excluded on the basis of age, sex or any other socio-demographic characteristic.

\section{Types of interventions}

We will include studies in which mLearning interventions were used to deliver the learning content of the course. This includes studies where mLearning methods were the sole means by which the intervention are delivered, or where mLearning methods were part of a complex, multi-component intervention (i.e., blended learning), as long as the contribution of mLearning component to overall learning has been assessed.

Only studies that compare an mLearning intervention to any form of traditional learning (i.e., any learning activity undertaken in the traditional classroom environment including face-to-face instruction, practical work or independent study), another form of mLearning or eLearning modalities will be considered eligible. "No intervention" as a comparison will be considered eligible only in studies on participants enrolled in a post-registration health professional educational programme.

As earlier noted, we will define mLearning interventions as any teaching, learning and or training intervention that is delivered through handheld mobile devices using wireless transmissions: third generation of mobile telecommunications technology (3G), fourth generation of mobile telecommunications technology (4G), global system for mobile communications, originally groupe spécial mobile (GSM), general packet radio services (GPRS), enhanced data rates for GSM evolution (EDGE or EGPRS), MMS, SMS, universal mobile telecommunications dystem (UMTS), wireless networking (wifi or any other wireless local area network (WLAN)) or long term evolution (LTE) standard.

Handheld mobile devices include but are not limited to mobile phones, smartphones, PDAs, phablets, tablets and Moving Picture Experts Group (MPEG)-1 audio layer 3 (MP3) players. If the mLearning was designed specifically to be used wirelessly i.e., 'no cord used' for content delivery and communication, laptops also meet our inclusion criteria. Many laptops have not just wireless but also LTE connectivity.

We will exclude any intervention using stationary technology, such as desktop computers. These will be considered in our "sister" reviews on offline and online computer based eLearning for health professional education.

\section{Types of outcome measures}

\section{Primary outcomes}

- Students' knowledge, measured using any validated or nonvalidated instrument to measure difference in pre- and post-test scores

- If several post-test results are available, we will record data as to when those tests were conducted and use the difference between the pre-test and the first post-test for the analysis

- When applicable, we will use the difference between the pre-test and the last-test available for sensitivity analysis (Sensitivity analysis)

- Students' skills, measured using any validated or nonvalidated instrument (e.g., pre- and post-test scores, time to perform a procedure, number of errors made whilst performing a procedure)

- Students' professional attitudes towards patients (e.g., awareness of moral and ethical responsibilities involved in patient contact) and/or towards new clinical knowledge or skills measured using only validated instruments

- Students' satisfaction with the learning intervention measured using only validated instruments

\section{Secondary outcomes}

- Patient related outcomes (only for interventions delivered to post-registration participants)

- Cost and cost-effectiveness of the intervention

- Adverse and/or unintended effects of mLearning (e.g., mortality, morbidity, medical errors etc.)

\section{Search methods for identification of studies}

\section{Electronic searches}

We will search the following databases:

- MEDLINE (via Ovid);

- EMBASE (via Elsevier); 
- the Cochrane Central Register of Controlled Trials (CENTRAL) (via Wiley);

- PsycINFO (via Ovid);

- Cumulative Index to Nursing and Allied Health Literature (CINAHL) (via EBSCOhost);

- Educational Resource Information Centre (ERIC) (via Ovid);

- Web of Science Core Collection (via Thomson Reuters)

We will define and use a common search strategy for all our Cochrane reviews in a series on eLearning for health professional education as they have been defined above in the Types of interventions section. We will not use a methodological filter and will screen references in multiple steps to ensure maximum sensitivity and specificity. Two independent authors (LTC, CKN) will conduct all steps of screening the references, who will in the first step of screening titles and abstracts put references into the above defined groups e.g., offline eLearning, online eLearning, mLearning. For any references where authors are unsure of categorisation or whether the study meets inclusion criteria, we will obtain a fulltext article to aid decision-making and ultimately use a third author as an arbiter where uncertainty remains.

\section{Searching other resources}

We will search reference lists of all included studies and relevant systematic reviews. We will also search the World Health Organization (WHO) International Clinical Trials Registry Platform (ICTRP) Search Portal (http://apps.who.int/trialsearch/) and the metaRegister of Controlled Trials (http://www.isrctn.com/page/ mrct) to identify unpublished trials and contact the relevant investigators for further information.

\section{Data collection and analysis}

\section{Selection of studies}

We will implement the search strategy as described in Electronic searches, and import all references identified to a reference management software. We will combine the search results from the different electronic databases and remove duplicate records. We plan to calibrate the screening of studies between the authors using the first 500 citations. Two authors (LTC, CKN) will independently screen titles and abstracts to identify studies that potentially meet inclusion criteria. We will retrieve the full-text articles of those articles. Finally, two authors (LTC, CKN) will independently assess the full text of the retrieved articles for compliance with our inclusion and exclusion criteria. We will resolve any disagreements through discussion between the two authors. If no agreement can be reached, we will consult a third author (JC). We will contact study investigators/authors in the case of unclear or missing information. We will list studies which appeared to be relevant but are excluded at this stage in the 'Characteristics of excluded studies' table with the reason for exclusion. Two authors (LTC, CKN) will verify the final list of included studies.

\section{Data extraction and management}

Two authors (LTC, CKN) will independently extract and manage the data for each of the included studies using a structured data recording form, derived from the data extraction template provided by the Cochrane Effective Practice and Organisation of Care (EPOC) Group (Cochrane EPOC Group 2015). We will pilot the data extraction form and amend it according to the received feedback. In addition to the usual information on study design and participants' demographics, we will extract data on other relevant fields, including type of device used, delivery method (e.g., email, SMS), type of content (e.g., video, text, images), and mode of mLearning (active or passive, linear or dynamic). We plan to contact study authors in the case of any unclear or missing information. We will resolve disagreements between review authors by discussion; we will consult a third review author (JC) in case disagreements cannot be resolved.

\section{Assessment of risk of bias in included studies}

Two authors (LKC, CKN) will independently assess the methodological quality of RCTs and cluster-RCTs using the Cochrane's tool for assessing risk of bias (Higgins 2011). We will pilot the risk of bias assessment between the reviewers and contact study authors in case of any unclear or missing information. We will assess risk of bias in included RCTs using the following domains: random sequence generation; allocation sequence concealment; blinding (participants, personnel); blinding (outcome assessment); completeness of outcome data, selective outcome reporting; and other sources of bias (e.g. baseline imbalance, inappropriate administration of an intervention and contamination). For cluster RCTs we will also assess the risk of these additional biases: recruitment bias; baseline imbalance; loss of clusters; incorrect analysis; and comparability with individually randomised trials. Judgements concerning risk of bias for each study will be classified using "yes ", "no" or "unclear", indicating high, low or unclear risk of bias respectively. We will incorporate the results of the risk of bias assessment into the review using a risk of bias table, graph and summary.

\section{Measures of treatment effect}

For continuous outcomes, we will calculate mean differences (MDs) and 95\% confidence intervals (CIs). For dichotomous outcomes, we will calculate risk ratios (RRs) and 95\% CIs. We will inflate the variances for clustering in cluster-RCTs, where we will 
record the cluster size, number of clusters and the intraclass correlation coefficient (ICC) (or estimate equivalent) for a study.

If more than one study measures the same outcome using different tools, we will recalculate MDs for each study into standardised mean differences (SMDs) by dividing the MD between groups by the standard deviation (SD) of outcome among participants.

\section{Unit of analysis issues}

For cluster-RCTs, we will attempt to obtain data at the student level. In the cases where the statistical analysis of the cluster-RCT has already been adjusted for the clustering of data, we will simply extract the reported effect estimates and use them directly for our analysis. In those cases where the individual data are not available in the study report, we will start by contacting the study investigators and authors to request these data and then meta-analyse them using a generic inverse-variance method in Cochrane statistical software, Review Manager 2014, which accounts for the clustering of data. When access to student-level data is not possible, we will extract a summary effect measurement for each cluster. We will consider the number of clusters as the sample size and our analysis will proceed as if the trial was individually randomised. It must be noted that this technique reduces the statistical power of analyses.

\section{Dealing with missing data}

We will contact the original study investigators for clarification or to request missing information. If we are unable to obtain this, we will use data available from the published studies and assess the risk of bias through the criterion 'incomplete outcome data'. We will not impute any missing data and will discuss all assumptions and subsequent procedures used to deal with missing values in the review. We will, where possible, conduct analysis on an intentionto-treat basis.

\section{Assessment of heterogeneity}

We will decide if it is appropriate to pool our measures of effect by assessing if the included studies are similar enough (in terms of their population, intervention characteristics, and reported outcomes) to make meaningful conclusions. If a meta-analysis of the included studies is indicated, we will assess statistical heterogeneity by visual inspection of the scatter of effect estimates in the forest plot and by calculating the $\mathrm{I}^{2}$ statistic (Higgins 2011), after using the inverse variance method. In case of a high degree of heterogeneity $\left(\mathrm{I}^{2}\right.$ greater than 0.5$)$, we will explore possible reasons for variability by conducting subgroup analysis.

Where we detect substantial clinical, methodological or statistical heterogeneity across included studies, we will not report pooled results from meta-analysis but will instead use a narrative approach to data synthesis. In this event we will attempt to explore possible clinical or methodological reasons for this variation by grouping studies that are similar in terms of populations, intervention features, methodological features, or other factors to explore differences in intervention effects.

\section{Assessment of reporting biases}

We will assess reporting bias qualitatively based on the characteristics of the included studies (e.g., if only small studies that indicate positive findings are identified for inclusion), and if information that we obtain from contacting experts and authors or studies suggests that there are relevant unpublished studies. If we include at least 10 studies, we will assess reporting bias using a funnel plot regression weighted by the inverse of the pooled variance. A regression slope of zero will be interpreted as absence of small study bias.

\section{Data synthesis}

We will report data using Review Manager 2014. We will enter extracted data into tables grouped by study design and type of intervention to create a descriptive synthesis. We will report the results of individual RCTs and cluster-RCTs as MDs for continuous variables and RRs for dichotomous variables with 95\% CIs. Using Miller's classification of clinical competence (Miller 1990), we will group the different types of tests for students' knowledge and skills and analyse them together. For example, we will analyse multiple choice questions assessing knowledge (i.e., 'knows') together and essay questions assessing competence (i.e., 'knows how') will be analysed together. The focus will therefore be on the testing method rather than the delivery method (i.e., if skills were assessed by a knowledge test, it would be categorised as knowledge).

For students' professional attitudes, we will group the different types of assessment and analyse them as cognitive attitudes, behavioural attitudes or affective attitudes as described by Martin 2002. Students' satisfaction will include the satisfaction and attitudes towards the learning intervention they were exposed to. We will assess students' professional attitudes and satisfaction narratively as available findings suggest that there is a high level of heterogeneity in the operational definition of these outcomes across studies (George 2014, Rasmussen 2014; WHO 2013) .

Where studies report more than one measure for each outcome, we will use the primary measure as defined by the primary study authors in the analysis. Where no primary measure has been reported, we will calculate and use a mean value of all the measures for the outcome in the analysis. The choice of model will depend on the level of heterogeneity between studies included in the meta-analysis (Assessment of heterogeneity). If meta-analysis is feasible, we will use a random-effects model, which provides a more conservative estimate of effect and can be used where there is moderate heterogeneity. We will separately report interventions for pre- and post-registration healthcare professionals. 
We will include the intention-to-treat analysis of the results in the meta-analysis.

\section{Subgroup analysis and investigation of heterogeneity}

We will conduct the following subgroup analyses (i.e., stratified analyses) in this study.

- Stratified by countries' income (low and middle income countries versus high income countries).

- Stratified by registration stage (pre- and post-registration interventions).

- Stratified by type of student or professional group (i.e., dental studies, medicine, nursing and midwifery, medical diagnostic and treatment technology, therapy and rehabilitation, and pharmacy).

- Stratified by number of repeated interventions (one-off versus repeated interventions).

- Stratified by type of handheld mobile devices used (e.g., mobile phones, tablets or PDAs).

- Stratified by adherence/time spent on the intervention (depending on the number of studies, per tertile, quartile or quantile). We will recalculate and present the measure of adherence/time spent on the intervention as a percentage to account for the different measures used across included studies.

- Stratified by delivery mode (i.e., exclusive mLearning versus blended learning).

We acknowledge that there are many other subgroup analyses that could be performed, for example comparing interventions according to learning objectives and interactivity of interventions. Future reviews, conducted after completion of our series of initial reviews, are in the best position to do this, because such comparisons would be most meaningful to an educator if multiple methods of eLearning were compared.

\section{Sensitivity analysis}

We will perform sensitivity analyses to explore the impact of the risk of bias dimensions on the outcomes of the review. We will remove studies deemed to be at high risk of bias from the analysis, after examination of individual study characteristics, to examine the effect on the pooled effects of the intervention.

We will exclude studies according to the following filters:

- high risk of bias studies (as specified above);
- smallest studies;

- source of funding (industry sponsorship (solely industryfunded), mixed sponsorship (public and industry funded, including free provision of study material only), non-industry sponsorship (solely public funded and no free provision of material), not described or unclear);

- time lapse between end of intervention and first post-test (quartiles) as well as last post-test.

For any studies comparing more than one mLearning or blended learning intervention to traditional learning, we will perform a sensitivity analysis to assess the impact of successively replacing the results of each intervention group on the measure of effect. Additionally, we will average the mean scores for each intervention group and use this average in the meta-analysis. We will then compare the difference between the two approaches.

\section{Summary of findings table}

We intend to prepare a 'Summary of findings' table to present the meta-analysis results, based on the methods described in chapter 11 of the Cochrane Handbook for Systematic Reviews of Interventions (Schünemann 2011). We will present the results of meta-analysis for the major comparisons of the review, for each of the major primary outcomes as well as potential adverse effects, as defined in the 'Types of outcome measures' section. We will provide a source and rationale for each assumed risk cited in the table(s). Two authors will use the GRADE criteria to rank the quality of the evidence using the GRADEprofiler (GRADEpro) software (Schünemann 2011). If meta-analysis is not feasible, we will present results in a narrative 'Summary of findings' table format, such as that used by Chan 2011 (CCCRG 2014).

\section{ACKNOW LEDGEMENTS}

We would like to thank the Cochrane Tobacco Addiction Group, in particular Dr Nicola Lindson-Hawley (Managing Editor), Ms Lindsay Stead and Ms Monaz Mehta for their support and guidance, as well as the UK Cochrane Centre for their workshops in Oxford. We would also like to thank Mr Carl Gornitzki, Ms GunBrit Knutssön and Mr Klas Moberg from the University Library, Karolinska Institutet for developing the search strategy. We gratefully acknowledge funding from the Lee Kong Chian School of Medicine, Nanyang Technological University eLearning for health professionals education grant. 


\section{R E F E R E N C E S}

\section{Additional references}

Ally 2009

Ally M. Mobile Learning: Transforming the Delivery of Education and Training. Athabasca University Press, 2009.

\section{Campbell 2013}

Campbell J, Dussault G, Buchan J, Pozo-Martin F, Guerra Arias M, Leone C, et al. A universal truth: no health without a workforce. Forum Report, Third Global Forum on Human Resources for Health, Recife, Brazil. Geneva, Global Health Workforce Alliance and World Health Organization, 2013. Available from http://www.who.int/ workforcealliance/knowledge/resources/hrhreport2013/en/.

\section{CCCRG 2014}

Cochrane Consumers and Communication Review Group. Standard Protocol Text and Additional Guidance for Review Authors. Available from http://cccrg.cochrane.org 2014.

\section{Chan 2011}

Chan RJ, Webster J, Marquart L. Information interventions for orienting patients and their carers to cancer care facilities. Cochrane Database of Systematic Reviews 2011, Issue 12. [DOI: 10.1002/14651858.CD008273.pub2]

\section{Chen 2010}

Chen LC. Striking the right balance: health workforce retention in remote and rural areas. Bulletin of the World Health Organization 2010;88(5):321-400.

\section{Childs 2005}

Childs S, Blenkinsopp E, Hall A, Walton G. Effective elearning for health professionals and students - barriers and their solutions. A systematic review of the literature - findings from the HeXL project. Health Information \& Libraries Journal 2005;22:20-32.

\section{Cochrane EPOC Group 2015}

Cochrane Effective Practice and Organisation of Care Group. Effective Practice and Organisation of Care (EPOC). EPOC Resources for review authors. Oslo: Norwegian Knowledge Centre for the Health Services; 2015. Available at: http://epoc.cochrane.org/epoc-specificresources-review-authors.

Cook 2010

Cook DA, Levinson AJ, Garside S, Dupras DM, Erwin PJ, Montori VM. Instructional design variations in internetbased learning for health professions education: a systematic review and meta-analysis. Academic Medicine 2010;85(5): $909-22$.

Crisp 2008

Crisp N, Gawanas B, Sharp I. Training the health workforce: scaling up, saving lives. Lancet 2008;371(9613):689-91.

\section{Crompton 2013}

Crompton H. A historical overview of mobile learning: Toward learner-centered education. In: Berge ZL, Muilenburg LY editor(s). Handbook of mobile learning. Florence, KY: Routledge, 2013:3-14.
Feng 2013

Feng JY, Chang YT, Chang HY, Erdley WS, Lin CH, Chang YJ. Systematic review of effectiveness of situated e-learning on medical and nursing education. Worldviews on EvidenceBased Nursing 2013;10(3):174-83.

\section{Frenk 2010}

Frenk J, Chen L, Bhutta ZA, Cohen J, Crisp N, Evans T, et al. Health professionals for a new century: transforming education to strengthen health systems in an interdependent world. Lancet 2010;376(9756):1923-58.

\section{George 2014}

George PP, Papachristou N, Belisario JM, Wang W, Wark PA, Cotic Z, et al. Online eLearning for undergraduates in health professions: A systematic review of the impact on knowledge, skills, attitudes and satisfaction. Journal of Global Health 2014;4(1):010406.

Global Health Workforce Alliance 2015

Global Health Workforce Alliance. About the Alliance. 2015. Available from http://www.who.int/ workforcealliance/about/en/.

\section{Hashemi 2011}

Hashemi M, Azizinezhad M, Najafi V, Nesari AJ. What is mobile learning? Challenges and capabilities. ProcediaSocial and Behavioral Sciences 2011;30:2477-481.

\section{Higgins 2011}

Higgins JPT, Green S (editors). Cochrane Handbook for Systematic Reviews of Interventions Version 5.1.0 [updated March 2011]. The Cochrane Collaboration, 2011. Available from www.cochrane-handbook.org.

\section{ITU 2015}

International Telecommunication Union (ITU). World Telecommunication/ICT Indicators database 2015 (19th Edition/June 2015). Available from http://www.itu.int/en/ ITU-D/Statistics/Pages/publications/wtid.aspx.

\section{Kuehn 2007}

Kuehn BM. Global shortage of health workers, brain drain stress developing countries. JAMA 2007;298(16):1853-5.

\section{Lahti 2014}

Lahti M, Hätönen H, Välimäki M. Impact of e-learning on nurses' and student nurses knowledge, skills, and satisfaction: a systematic review and meta-analysis. International Journal of Nursing Studies 2014;51(1):136-49.

Marchal 2003

Marchal B, Kegels G. Health workforce imbalances in times of globalization: brain drain or professional mobility?. International Journal of Health Planning and Management 2003;18(S1):S89-S101.

\section{Martin 2002}

Martin J, Lloyd M, Singh S. Professional attitudes: can they be taught and assessed in medical education?. Clinical Medicine 2002;2:217-23. 


\section{Miller 1990}

Miller GE. The assessment of clinical skills/competence/ performance. Academic Medicine 1990;65(9):S63-7.

Motiwalla 2007

Motiwalla LF. Mobile learning: A framework and evaluation. Computers \& Education 2007;49(3):581-96.

O’Malley 2005

O’Malley C, Vavoula G, Glew JP, Taylor J, Sharples M, Lefrere P, et al. Guidelines for learning/teaching/tutoring in a mobile environment. Public deliverable from the MOBILearn project (D.4.1). 2005. Available from https:// hal.archives-ouvertes.fr/hal-00696244.

\section{OECD 2001}

Organisation for Economic Cooperation and Development. Understanding the Digital Divide. Jan 2001. Available from http://www.oecd-ilibrary.org/science-and-technology/ understanding-the-digital-divide_236405667766.

\section{Ozdamli 2011}

Ozdamli F, Cavus N. Basic elements and characteristics of mobile learning. Procedia-Social and Behavioral Sciences 2011;28:937-42.

Pew Research Center 2014

Pew Research Center. Mobile Technology Fact Sheet. 2014. Available from http://www.pewinternet.org/fact-sheets/ mobile-technology-fact-sheet/.

Rasmussen 2014

Rasmussen K, Belisario JM, Wark PA, Molina JA, Loong SL, Cotic Z, et al. Offline eLearning for undergraduates in health professions: A systematic review of the impact on knowledge, skills, attitudes and satisfaction. Journal of Global Health 2014;4(1):010405.

Review Manager 2014

The Nordic Cochrane Centre, The Cochrane Collaboration. Review Manager (RevMan). 5.3. Copenhagen: The Nordic Cochrane Centre, The Cochrane Collaboration, 2014.

\section{Rosenberg 2003}

Rosenberg H, Grad HA, Matear DW. The effectiveness of computer-aided, self-instructional programs in dental education: a systematic review of the literature. Journal of Dental Education 2003;67(5):524-32.

Rowe 2012

Rowe M, Frantz J, Bozalek V. The role of blended learning in the clinical education of healthcare students: A systematic review. Medical Teacher 2012;34(4):e216-21.

Sangrà 2012

Sangrà A, Vlachopoulos D, Cabrera N. Building an inclusive definition of e-learning: An approach to the conceptual framework. International Review of Research in

Open and Distance Learning 2012;13(2):145-59.

\section{Schünemann 2011}

Schünemann HJ, Oxman AD, Higgins JPT, Vist GE, Glasziou P, Guyatt GH. Chapter 11: Presenting results and 'Summary of findings' tables [Cochrane Handbook for Systematic Reviews of Interventions Version 5.1.0 (updated March 2011)]. In: Higgins JPT, Green S (editors) editor (s). Available from www.cochrane-handbook.org. WileyBlackwell, 2011.

\section{Sharples 2002}

Sharples M. Disruptive devices: mobile technology for conversational learning. International Journal of Continuing Engineering Education and Life Long Learning 2002;12(5): 504-20.

\section{Trifonova 2003}

Trifonova A, Ronchetti M. Where is Mobile Learning Going?. Proceedings of World Conference on E-Learning in Corporate, Government, Healthcare, and Higher Education. 2003:1794-1801.

\section{UIS 2012}

UNESCO Institute for Statistics. International Standard Classification of Education (ISCED) 2011. December 2012. Available from http://www.uis.unesco.org/ Education/Pages/international-standard-classification-ofeducation.aspx.

\section{WHO 2011}

World Health Organization. Transformative scale up of health professional education. An effort to increase the numbers of health professionals and to strengthen their impact on population health. March 2011. WHO reference number: WHO/HSS/HRH/HEP/2011.01. Available from http://www.who.int/hrh/resources/transformative_ education/en/.

\section{WHO 2013}

World Health Organization. Research for universal health coverage: World health report 2013. August 2013. Available from http://www.who.int/whr/2013/report/en/.

\section{World Bank 2013}

World Bank. World Development Indicators 2013. Washington, DC: World Bank. doi: 10.1596/978-0-82139824-1. License: Creative Commons Attribution CC BY 3.0. Available from http://databank.worldbank.org/data/ download/WDI-2013-ebook.pdf.

* Indicates the major publication for the study 


\section{A P P E N D I C ES}

\section{Appendix I. MEDLINE (Ovid) search strategy}

1. exp education, professional/ not education, veterinary/

2. Education, Predental/

3. Education, Premedical/

4. exp Students, Health Occupations/

5. ((medic* or premedic* or dent* or laborator* or predent* or midwi? e* or nurs* or nutrition* or orthop* or podiat* or pharmac* or psycholog* or psychiatr* or health or healthcare or occupational therap* or physiotherap* or physical therap* or clinical or surg* or $^{*}$ radiolog* or obstetric* or gyn?ecolog* or orthodont* or An?esthesi* or Dermatolog* or Oncolog* or Rheumatolog* or Neurolog* or Patholog* or P? ediatric* or Cardiolog* or Urolog*) adj3 (student* or graduate* or undergraduate* or staff or personnel or practitioner* or clerk* or fellow* or internship* or residen* ${ }^{*}$ or educat* or train* or novice* or tutor*)).tw,kf.

6. or/1-5

7. Computer-Assisted Instruction/

8. exp Internet/

9. Computer Simulation/

10. Patient Simulation/

11. software/

12. Mobile Applications/

13. User-Computer Interface/

14. Video Games/

15. Web Browser/

16. Education, Distance/

17. Computers/

18. exp Microcomputers/

19. exp Cell Phones/

20. Games, Experimental/

21. exp Models, Anatomic/

22. Audiovisual Aids/

23. Educational Technology/

24. Electronic Mail/

25. exp Telemedicine/

26. Telenursing/

27. Telecommunications/

28. Webcasts/

29. exp Videoconferencing/

30. ((computer* or digital* or hybrid or blended or mixed mode or distance or remote* or electronic or mobile or online* or interactiv* or multimedia or internet or web* or virtual* or game* or gaming or Videogame* or Videogaming) adj3 (classroom* or course* or

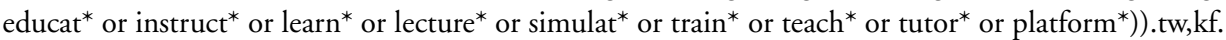

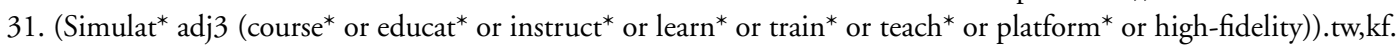

32. e-learn*.tw,kf.

33. elearn*.tw,kf.

34. m-learn*.tw,kf.

35. mlearn*.tw,kf.

36. smartphone*.tw,kf.

37. smart-phone*.tw,kf.

38. ((mobile or cell) adj2 phone*).tw,kf.

39. iphone*.tw,kf.

40. android*.tw,kf.

41. ipad*.tw,kf.

42. Personal digital assistant*.tw,kf.

Mobile learning for delivering health professional education (Protocol)

Copyright (๑) 2015 The Cochrane Collaboration. Published by John Wiley \& Sons, Ltd. 
43. handheld computer*.tw,kf.

44. Mobile App?.tw,kf.

45. Mobile Application?.tw,kf.

46. webcast*.tw,kf.

47. webinar*.tw,kf.

48. flipped classroom*.tw,kf.

49. Serious game*.tw,kf.

50. Serious gaming.tw,kf.

51. Patient Simulat*.tw,kf.

52. Virtual patient*.tw,kf.

53. ((educat* or instruct* or learn* or simulat* or train* or teach* or interactiv*) adj2 technolog*).tw,kf.

54. Massive Open Online Course?.tw,kf.

55. Mooc?.tw,kf.

56. (Canvas network or Coursera or Coursesites or edx or Futurelearn or iversity or miriada $\mathrm{x}$ or moodle or novoed or openlearning or open2study or plato or spoc or udacity or pingpong).tw,kf.

57. or/7-56

58. 6 and 57

59. Education.fs.

60. Education/

61. Teaching/

62. Learning/

63. exp Inservice Training/

64. Curriculum/

65. educat*.tw,kf.

66. learn*.tw,kf.

67. train*.tw,kf.

68. instruct*.tw,kf.

69. teach*.tw,kf.

70. or/59-69

71. Health Personnel/

72. exp Allied Health Personnel/

73. Anatomists/

74. "Coroners and Medical Examiners"/

75. exp Dental Staff/

76. exp Dentists/

77. Health Educators/

78. Infection Control Practitioners/

79. Medical Laboratory Personnel/

80. exp Medical Staff/

81. exp Nurses/

82. exp Nursing Staff/

83. Personnel, Hospital/

84. Pharmacists/

85. exp Physicians/

86. Physician*.tw,kf.

87. Doctor*.tw,kf.

88. Nurs*.tw,kf.

89. Surg*.tw,kf.

90. Health Personnel.tw,kf.

91. healthcare professional* ${ }^{*}$ tw,kf.

92. radiolog*.tw,kf.

93. dentist*.tw,kf.

94. Pharmacist*.tw,kf.

Mobile learning for delivering health professional education (Protocol)

Copyright $\odot 2015$ The Cochrane Collaboration. Published by John Wiley \& Sons, Ltd. 
95. Hospital Administrator*.tw,kf.

96. Podiatr*.tw,kf.

97. Psycholog*.tw,kf.

98. Psychiatr*.tw,kf.

99. An?esthesi*.tw,kf.

100. Clinician*.tw,kf.

101. Dermatolog*.tw,kf.

102. General practioner*.tw,kf.

103. Cardiolog*.tw,kf.

104. Oncolog*.tw,kf.

105. Rheumatolog*.tw,kf.

106. Neurolog*.tw,kf.

107. Patholog*.tw,kf.

108. P?ediatric*.tw,kf.

109. Physiotherap*.tw,kf.

110. Physical therap*.tw,kf.

111. Occupational therap*.tw,kf.

112. dieti?ian*.tw,kf.

113. Dietetic*.tw,kf.

114. midwi? $e^{*}$.tw,kf.

115. nutrition*.tw,kf.

116. orthopti*.tw,kf.

117. obstetric*.tw,kf.

118. gyn?ecolog*.tw,kf.

119. orthodont*.tw,kf.

120. Urolog*.tw,kf.

121. or/71-120

122. Health Occupations/

123. exp Allied Health Occupations/

124. Biomedical Engineering/

125. Chiropractic/

126. exp Dentistry/

127. exp Evidence-Based Practice/

128. exp Medicine/

129. exp Nursing/

130. Dietetics/

131. Optometry/

132. Orthoptics/

133. exp Pharmacology/

134. exp Pharmacy/

135. Podiatry/

136. Psychology, Medical/

137. Serology/

138. Specialization/

139. exp Surgical Procedures, Operative/

140. exp Radiography/

141. or/122-140

142. 121 or 141

143. 57 and 70 and 142

144. Psychomotor Performance/

145. motor skills/

146. ((psychomotor or procedural or technical) adj3 skill*).tw,kf.

147. (psychomotor adj3 performance).tw,kf.

Mobile learning for delivering health professional education (Protocol)

Copyright $\odot 2015$ The Cochrane Collaboration. Published by John Wiley \& Sons, Ltd. 
148. or/144-147

149. 6 and 148

150.58 or 143 or 149

151. limit 150 to $\mathrm{yr}=" 1990$-Current“

\section{CONTRIBUTIONS OFAUTHORS}

JC conceived the idea for the review. ERS and JMB wrote the protocol. LTC peer-reviewed it and addressed the reviewers comments. $\mathrm{CKN}, \mathrm{AM}, \mathrm{NZ}$ and JC provided comments on the protocol.

\section{DECLARATIONSOF INTEREST}

None known.

\section{SOURCES OF SUPPORT}

\section{Internal sources}

- Imperial College London, UK.

- NTU Lee Kong Chian School of Medicine, Singapore.

- Karolinska Institutet, Sweden.

- The Health Services and Outcomes Research (HSOR) National Healthcare Group, Singapore.

\section{External sources}

- No sources of support supplied 